\title{
The biology and evolution of polyspermy: insights from cellular and functional studies of sperm and centrosomal behavior in the fertilized egg
}

\author{
Rhonda R Snook, David J Hosken ${ }^{1}$ and Timothy L Karr ${ }^{2}$ \\ Department of Animal and Plant Sciences, University of Sheffield, Sheffield, S10 2TN, UK, ${ }^{1}$ School of Biosciences, \\ Centre for Ecology and Conservation, University of Exeter, Cornwall Campus, Penryn, Cornwall, TR10 9EZ, UK and \\ ${ }^{2}$ Biodesign Institute, Center for Infectious Diseases and Vaccinology, Evolutionary Functional Genomics, Arizona \\ State University, Tempe, Arizona 85287-5401, USA
}

Correspondence should be addressed to R R Snook; Email: r.snook@sheffield.ac.uk

\begin{abstract}
Recent studies of centrosome biogenesis, microtubule dynamics, and their management point to their role in mediating conditions such as aging and cancer. Centrosome dysfunction is also a hallmark of pathological polyspermy. Polyspermy occurs when the oocyte is penetrated by more than one sperm and can be pathological because an excess of centrosomes compromises development. However, in some taxa, multiple sperm enter the egg with no apparent adverse effect on zygote viability. Thus, some taxa can manage excess centrosomes and represent cases of non-pathological polyspermy. While these two forms of polyspermy have long been known, we argue that there is limited understanding of the proximate and ultimate processes that underlie this taxonomic variation in the outcome of polyspermy and that studying this variation could help uncover the control and role(s) of centrosomes during fertilization in particular, but also mitosis in general. To encourage such studies we: 1) describe taxonomic differences in the outcome of polyspermy, 2) discuss mechanistic aspects of reproductive biology that may contribute to the different consequences of polyspermy, and 3) outline the potential selective events that could lead to the evolution of variation in polyspermy outcomes. We suggest that novel insights into centrosome biology may occur by cooperative studies between reproductive and evolutionary biologists focusing on the mechanisms generating variation in the fitness consequences of polyspermy, and in the taxonomic distribution of all these events. The consequent discoveries of these studies may lead to informative insights into cancer and aging along with other centrosome-related diseases and syndromes. Reproduction (2011) 142 779-792
\end{abstract}

\section{Introduction}

Polyspermy, the penetration of the oocyte cytoplasm by more than one spermatozoon (Hunter 1998), is one of the most commonly observed 'abnormalities' in fertilization, generally resulting in embryonic death (Boveri 1901, Wang et al. 2003). The pathological effect of polyspermy is due to mismanagement of the centrosomes. The centrosome consists of two components; a pair of orthogonally arranged centrioles and a surrounding pericentriolar material (PCM) containing several proteins. The centrioles, in combination with the PCM, control microtubule organization and thus the centrosome is also known as the microtubule-organizing center (MTOC). With respect to development, the sperm centrosome typically (but not always) organizes the first mitotic spindle via a sperm aster so a functioning centrosome is vital for a number of developmental processes - or at least their timing (Karr 2001, Basto et al. 2006, 2008, Schatten 2008). However, only one is needed and, if there is more than one active centrosome in the zygote, then developmental anomalies can occur. For example, an excess of centrosomes can result in multipolar spindles that cause disruptions in chromosome dynamics during mitosis. Therefore, the activity of centrosomes arising from supernumerary sperm must be tightly regulated to prevent cell death (Karr 2001).

Any pathological effects of polyspermy on development (Hunter 1998) should result in selection against multiple sperm penetration of the ovum, with females investing in mechanisms that allow them to 'resist' polyspermy. That is, to limit the number of sperm entering the egg. A number of such mechanisms have evolved, with the best understood being the fast and slow blocks to polyspermy (see Wong \& Wessel (2005) for a review). Intriguingly, however, a number of species can 'tolerate' polyspermy, having supernumerary sperm enter the egg without any pathological effects (Rothschild 1956, Elinson 1986, Tarín 2000). 
In taxa exhibiting pathological polyspermy, each supernumerary sperm forms a sperm aster, each functioning like a centrosome, generating multiple MTOCs and subsequently, developmental problems. For polyspermy to be non-pathological, one would predict that each supernumerary sperm fails to form a sperm aster. However, this is not the case; all sperm form an aster but only one sperm acts as a functional centrosome (Elinson 1986, Carré et al. 1991, Long et al. 1993, Linhart \& Kudo 1997, Stepinska \& Bakst 2007). Thus, at a first approximation, the differences in the consequences of polyspermy appear to relate to the (mis)management of centrosomes that give rise to multiple MTOCs. What remains unknown are the mechanisms that mitigate the effects of supernumerary sperm-derived centrioles in some, but not other, taxa. What are the mechanistic differences between resistance and tolerance to polyspermy? Why does this variation exist? What are the evolutionary causes and consequences of this variation?

Answering these questions goes beyond understanding fertilization and the effects of polyspermy. Centrosome biology is becoming an increasingly important area of research given that supernumerary centrosomes and the attendant abnormal mitoses caused by them are associated with cancer and aging (reviewed in Schatten (2008)). Thus, the study of fertilization, polyspermy and zygote formation in the context of centrosome management may provide important insights into general cellular biology and disease progression. In this study, we argue that studying mechanisms that contribute to the taxonomic variation in the outcome of polyspermy may represent a hitherto unexploited pathway to understanding centrosome biology.

To support this supposition, we discuss aspects of the reproductive biology of taxa displaying either pathological or non-pathological polyspermy to ascertain the reproductive, cellular, and developmental traits that potentially explain variation in these two outcomes of polyspermy. We first provide a general overview of interspecific variation in polyspermy, relating this to developmental outcomes and associated reproductive, cellular, and developmental traits. We then consider the evolutionary causes and consequences of variation in the outcome of polyspermy across taxa.

\section{Variation in the outcome of polyspermy \\ Pathological polyspermy}

\section{Description and taxonomic distribution}

Pathological polyspermy occurs across a wide taxonomic range, including both vertebrates and invertebrates, and external and internal fertilizers (see Schatten (1994) and Tarín (2000) for review). Blocks to polyspermy have evolved that limit the number of sperm entering the egg, including the well-studied fast and slow blocks to polyspermy (see Tarín (2000) and
Wong \& Wessel (2005) for reviews). While very effective in enforcing monospermy, these blocks are not perfect and polyspermy can still occur. When polyspermy does occur, chromosome dynamics are negatively affected because each sperm brings in a basal body that can generate an active centrosome and MTOC resulting in the formation of multiple (supernumerary) sperm asters.

\section{Supernumerary sperm aster dynamics}

Pathological polyspermy has been somewhat studied, although mostly as a byproduct of understanding normal fertilization biology. Thus, extensive knowledge about how supernumerary sperm are (mis)managed and the molecular characterization of centrosome formation during fertilization are described in relatively few taxa (Breed et al. 1994, Navara et al. 1994, Simerly et al. 1999, Sun \& Schatten 2007). Space limitation does not permit us to review this extensive literature and so here we provide a very brief review of sperm aster formation and the subsequent fitness consequences for some pathologically polyspermic taxa for general comparison with centrosome dynamics in taxa exhibiting non-pathological polyspermy.

Taxa vary in whether most supernumerary sperm asters fuse with the egg nucleus, with each other, or whether each remains separate (barring the one which fuses with the egg nucleus). For example, in sea urchins (echinoderm), two or more sperm pronuclei may fuse with the egg pronucleus (other sperm nuclei may remain separate in the egg cytoplasm and do not fuse with each other). This multiple fusion results in a multipolar (usually quadripolar) spindle subsequently dividing simultaneously into four blastomeres (Fig. 1a). The chromosomes on these multipolar spindles are distributed at random and irregularly to all poles. These multiple asters can form aberrant mitotic spindles that appear to compete for available chromosome binding sites (presumably kinetochore binding sites). This can lead to subsequent chromosome fragmentation, resulting from competing forces generated during mitosis, manifesting as blastomeres that contain different and usually incomplete chromosome sets (Laubichler \& Davidson 2008). The resulting pathological effect on development varies depending on the extent to which the chromosomes are unbalanced (Fig. 1a).

In other pathologically polyspermic taxa, such as Xenopus frogs, each sperm, including the one fusing with the egg pronucleus, gives rise to a bipolar spindle each of which subsequently cleaves. Thus, the number of cleavages is equal to the number of sperm entering the egg. Each blastomere can vary in ploidy, however, depending on how the chromosomes are affected (Fig. 1b). Some will contain the normal diploid chromosomal content deriving from the first embryonic nucleus, whereas others will contain only a haploid chromosomal content derived from the supernumerary 

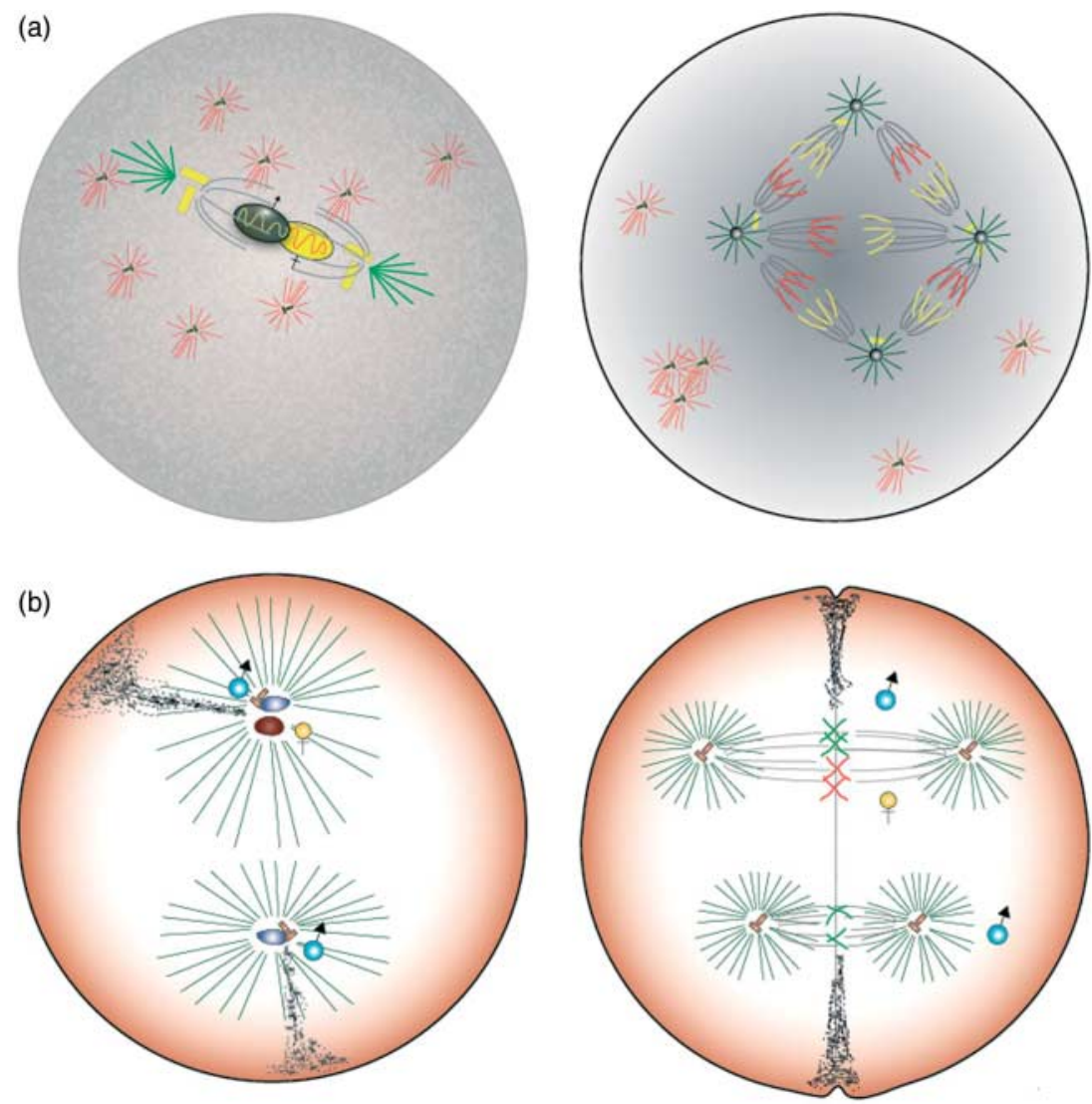

Figure 1 Pathological polyspermy in echinoderms and vertebrate taxa. (a) Polyspermy in a sea urchin egg containing eight supernumerary sperm asters. Left panel: supernumerary sperm asters (indicated by the red lines associated with the black ' $\mathrm{T}$ ' structure representing a centriole) migrate to form clusters in close apposition to the forming first mitotic spindle (microtubules in green and centrioles in paired yellow rectangles). Right panel: some of the supernumerary sperm asters successfully compete for mitotic chromosomes resulting, in this example, four mitotic aster poles. The ensuing mitosis results in aneuploid and polyploid nuclei and subsequent developmental failure of the embryo. Adapted from Wilson EB 1925 The Cell in Development and Inheritance. New York: Macmillan. (b) Diagram of polyspermy in the frog egg. Left panel: in this example, fertilization by three spermatozoa result in normal first mitosis resulting from the apposition and fusion of a male and female pronucleus (upper aster) while two sperm nuclei (lower aster) begin formation of a haploid mitotic figure. Microtubules in green and centrioles in paired yellow structures. Right panel: the resulting mitotic divisions and cleavage results in two cells containing $3 \mathrm{~N}$ chromosomal content. Continued cell divisions are often asynchronous and aneuploid presumably due to this chromosomal imbalance, which results in aberrant development and ultimately in embryonic death. Adapted from Wilson EB 1925 The Cell in Development and Inheritance. New York: Macmillan.

sperm. Such irregular cleavage results in aberrant development of varying degrees (Fig. 1b).

Thus, failure to manage extra centrosomes severely negatively impacts zygote formation and development, regardless of whether supernumerary sperm fuse with the egg pronucleus or generate their own bipolar spindle. This outcome is in stark contrast to the few taxa that have been studied which exhibit nonpathological polyspermy.

\section{Non-pathological and 'compensable' polyspermy}

\section{Description}

In many taxa, polyspermy is a regular occurrence yet has no apparent detrimental effect on subsequent embryonic development; historically this has been referred to as physiological polyspermy. In physiological polyspermy, supernumerary sperm always enter the egg. This is intriguing from a mechanistic standpoint as, like pathological polyspermy, each sperm forms a sperm aster upon entry into the egg. However, despite the production of multiple sperm asters, only a single bipolar spindle forms around the female pronucleus and one male pronucleus, with subsequent karyogamy (fusion of the nuclear material) and the first zygotic cleavage occurring normally (Rothschild 1956, Elinson 1986, Tarín 2000).

Here, we describe another type of non-pathological polyspermy that we term 'compensable polyspermy'. Compensable polyspermy describes the situation in which taxa normally exhibit monospermy, but when polyspermy occasionally occurs, no apparent negative consequences to embryonic development are observed. 
We refer to it as compensable because one would predict that polyspermy would be pathological given that fertilization is normally monospermic, yet the potentially negative effects of polyspermy are somehow compensated to ensure that only one single diploid zygote is produced. Like physiological polyspermy, in taxa where compensable polyspermy has been described, only a single sperm pronucleus fuses with the female pronucleus and the remaining sperm, while forming sperm asters, do not participate in fusion or cleavage. Compensable polyspermy is even less studied than the other types of polyspermy.

One practical problem with classifying taxa as exhibiting compensable polyspermy is that it is difficult to assess whether an egg has multiple sperm in it while concurrently determining that the ovum/embryo develops normally (given that polyspermy is normally seen in fixed samples where the developmental rate and fate of embryos cannot simultaneously be determined). Nonetheless, evidence consistent with compensable polyspermy being non-pathological includes observations of late-stage embryos having multiple sperm within them (Riemann 1965, Snook \& Karr 1998, Landmann et al. 2009, Riparbelli \& Callaini 2010), although whether polyspermy has some subsequent negative fitness consequences such as delaying development or reducing viability (Basto et al. 2006) cannot be determined. Ascertaining both the taxonomic distribution, and/or potential fitness consequences of, compensable polyspermy is an area requiring additional research.

Compensable polyspermy may simply represent part of a polyspermy continuum, but since physiological polyspermy has historically been used for species in which supernumerary sperm are always present, we distinguish between these two types of non-pathological polyspermy. Moreover, from both a developmental and evolutionary perspective, it may be profitable to view physiological and compensable polyspermy as different phenomena. In physiological polyspermy, the egg must always deal with multiple sperm and their functional centrosomes, whereas in compensable polyspermy the egg only occasionally encounters multiple sperm with functional centrosomes (similar to pathological polyspermy), yet there is no obvious detrimental developmental effects (unlike pathological polyspermy). This continuum provides considerable experimental potential to study centrosome biogenesis and management.

\section{Taxonomic distribution}

Non-pathological polyspermy has been described in numerous invertebrates taxa including arachnids (spiders; uncited reference in Iwamatsu (2000) page 119) and insects (e.g. various Diptera including Drosophila, Musca, and Calliphora, Orthopterans such as Gryllus, Coleopterans such as the rice weevil, and Collembola; Tiegs \&
Murray 1938, Riemann 1965, Degrugillier \& Leopold 1976, Jura \& Krzysztofowicz 1992, Calliani \& Riparbelli 1996, Snook \& Karr 1998, Sato \& Tanaka-Sato 2002), and vertebrates including elasmobranchs (sharks, skates, and rays; Rückert (1899) as cited in Rothschild (1956)), urodeles (newts and salamanders; Jordan (1893) as cited in Rothschild (1956)), reptiles (Oppel (1892) as cited in Rothschild (1956)), and birds (Blount 1909). All these taxa have internal fertilization and possess yolky eggs. One exception to this is the externally fertilizing ctenophore, Beroe ovata (Carré \& Sardet 1984, Carré et al. 1991). Taxa exhibiting non-pathological polyspermy have no obvious fast block to polyspermy (Tarín 2000, Wong \& Wessel 2005) and while some very slow blocks may exist (e.g. the formation of the outer perivitelline layer in birds; Karr et al. (2009)), they restrict, only to some degree, the number of supernumerary sperm entering the ovum.

Many insects were originally considered to exhibit physiological polyspermy (Huettner 1924, Counce 1959) given that there was a large amount of sperm material seen in eggs. However, subsequent work conclusively determined that typically only one sperm entered the egg (Hildreth \& Lucchesi 1963, Karr 1991, 1996, Karr \& Pitnick 1996, Snook \& Karr 1998). Although monospermy is now known to be a major mode of fertilization in insects, there are numerous examples of bona fide physiological polyspermy in insects (e.g. stick insects (Mantovani \& Scali 1992), crickets (Sato \& Tanaka-Sato 2002), and Collembola (Jura \& Krzysztofowicz 1992)). For example, in the cricket, Gryllus bimaculatus, there is a ring of eight micropyles (extensions of the chorion where sperm enter the egg) and 3-5 sperm are usually observed inside the egg. Only one sperm enters the tubulin-rich 'cytoplasmic island' on the egg dorsal side, and subsequently the egg nucleus, after completing meiosis near the ventral surface, moves toward the male pronucleus of this sperm to unite with it (Sato \& Tanaka-Sato 2002). The fate of the supernumerary sperm is unknown.

Compensable polyspermy has been described in insects, such as Drosophila (Snook \& Karr 1998). The extent of polyspermy ranges from 1 to $6 \%$ depending on the species and population (Karr 1991, Calliani \& Riparbelli 1996, Snook \& Karr 1998). In one extreme example, at least six sperm were seen to have entered the egg with embryonic development having proceeded at least through to the gastrula stage (Snook \& Karr 1998). This advanced developmental stage suggests that polyspermy does not hinder initial development. In Drosophila, supernumerary sperm replicate their DNA and undergo mitosis, having anaphase A like chromosome configurations and centrosome pairs at their poles (Calliani \& Riparbelli 1996, Calliani et al. 1999). These supernumerary sperm arrest at the meta/anaphase transition of the first mitosis and persist at least until the blastoderm stage (Riparbelli \& Calliani 2010); they 
do not appear to negatively impact development (Snook \& Karr 1998, Riparbelli \& Calliani 2010). In contrast, during anaphase $\mathrm{A}$ the spindles of the male and female pronuclei had only one centrosome for each pole during mitosis as expected (Calliani \& Riparbelli 1996). How the egg deals with supernumerary sperm and their attendant extra centrosomes is a conundrum. However, the centrosome duplication observed arising from supernumerary sperm (but without concomitant chromosome replication) suggests that at least some aspects of centrosome dynamics are active during this time period and that mechanisms may be in place to contain them (Calliani et al. 1999).

\section{Supernumerary sperm aster dynamics}

Despite the potential to use taxa exhibiting either physiological or compensable polyspermy to elucidate how multiple centrosomes can be managed without developmental abnormality, there is limited information on the mechanisms for centrosome control. In both physiological polyspermic urodeles and birds, supernumerary sperm asters are shunted away from the female pronucleus by unknown mechanisms, although there is variation between these taxa in the movement of extra sperm nuclei and possibly how they are degraded (Elinson 1986, Iwao 2000, Wishart \& Horrocks 2000). Rothschild (1956), in his landmark paper on polyspermy, suggested that in physiological polyspermy (what he called type II inhibition), some substance diffuses out of either the female nucleus or the uniting sperm nucleus to cause degeneration of supernumerary sperm.

In the newt, Cynops pyrrhogaster (urodele), supernumerary sperm move from the animal hemisphere to the vegetal hemisphere. The principle sperm nucleus - the one destined to fuse with the female pronucleus - develops the larger sperm aster and enters and exits metaphase earlier than the other sperm nuclei (Iwao et al. 2002; Fig. 2). Supernumerary sperm form monopolar spindles, then degrade before any cell division. Data suggest a sperm maturation-promoting factor (MPF) present in the egg acts to differentially 'mark' supernumerary sperm and this subsequently results in supernumerary sperm degradation (Iwao et al. 2002).

In birds, up to 60 sperm can enter the germinal disk, the preferential site of initial sperm-egg interactions (Perry 1987). A few hours after ovulation, two pronuclei, presumably one derived from the female and one from the male, can be observed paired in approximately the center of the germinal disk, with supernumerary sperm pronuclei moved to the disk periphery into the extraembryonic region (Wong \& Wessel 2005; Fig. 3). Some of the peripheral sperm asters may undergo one or two mitotic divisions, but subsequently degenerate at an early cleavage stage (Waddington et al. 1998, Wishart \& Horrocks 2000). These mitotic divisions, which presumably require a spindle and thus an MTOC, do not appear to interfere with normal development, unlike in taxa exhibiting pathological polyspermy. However, several reports have linked supernumerary sperm with aberrant development in birds (Fechheimer 1981, Forstmeier \& Ellegren 2010), although it is difficult to know whether these eggs were compromised in other ways. A recent study on zebra finch birds (Taeniopygia guttata) found evidence of chromosomal defects representing either maternal or paternal trisomies or triploidies in $1.4 \%$ of embryos examined; $17.6 \%$ of these abnormalities had paternal triploid origin, indicating either fertilization by diploid sperm or dispermy. Assuming these are the sole result of dispermy, then the likelihood of developmental
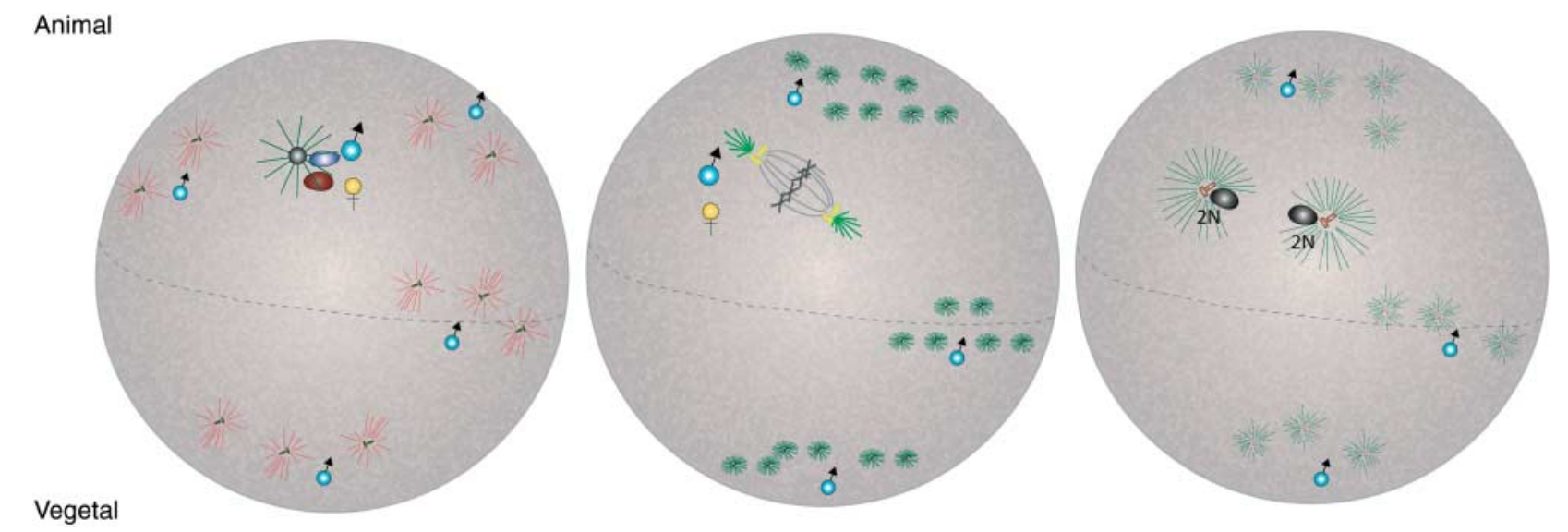

Figure 2 Physiological polyspermy in newts. Diagram of sperm aster formation in polyspermic urodele eggs. In this example, ten supernumerary sperm asters form throughout the fertilized egg (left; indicated by the red lines associated with the black ' $\mathrm{T}$ ' structure representing a centriole) surrounding the migrating male and female pronuclei indicated by the larger sperm aster surrounding the male pronucleus destined to fuse and form the zygote nucleus. Sperm aster growth, DNA replication and mitotic spindle formation (center) follows, but generally only the forming zygote arising from the male and female pronuclei complete mitosis (larger spindle) while all mitotic figures arising from the supernumerary sperm (smaller spindles) degenerate and do not participate further in zygote development. Adapted from Wilson EB 1925 The Cell in Development and Inheritance. New York: Macmillan. 


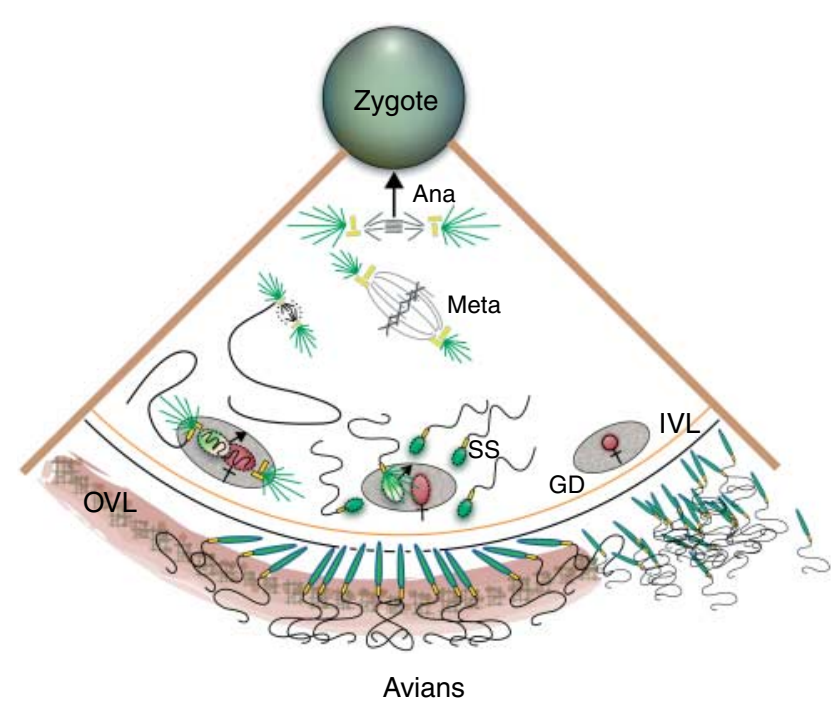

Figure 3 Physiological polyspermy in avian taxa. Diagram of avian fertilization. In the diagram, fertilization and development events proceed in a clockwise manner, sperm first encounter the inner vitelline layer (IVL) directly on the egg surface immediately following ovulation as the egg is enveloped by the infundibulus of the female's reproductive tract. Physiological polyspermy occurs preferentially in the area of the germinal disk (GD) where the female meiotic products reside. Soon after contact with the IVL, an outer vitelline layer (OVL) is quickly assembled trapping many sperm and preventing additional sperm entry. A single sperm enters the GD and fuses with the female pronucleus and the supernumerary sperm (SS) degenerate. The nature of karyogamy, nuclear fusion and chromosome behavior are less well known. Adapted, with permission, from Karr TL Swanson WJ \& Snook RR 2009 The evolutionary significance of variation in sperm-egg interactions. In Sperm Biology: An Evolutionary Perspective, pp 305-365. Eds TR Birkhead,

DJ Hosken \& S Pitnick. London: Academic Press. (C) 2009 Elsevier.

pathology as a consequence of physiological polyspermy is estimated to be ca. $0.2 \%$ of fertilized eggs. In reality, it is likely to be much less, as two studies have found that the frequency of aberrant development may be associated with individual mating pairs, or at least the female, in some investigations (Miller et al. 1976, Forstmeier \& Ellegren 2010).

In birds, supernumerary sperm may be degraded as a result of DNase activity in the oocyte (Stepinska \& Olszanska 2003) and this appears to be restricted to the egg cytoplasm next to the germinal disk (Stepinska \& Bakst 2007). The male and female pronuclei are possibly protected from DNase damage by associating only with the nucleoplasm, rather than the cytoplasm, within the oocyte (Stepinska \& Olszanska 2003). No such DNase activity has been found in the pathologically polyspermic mouse (Stepinska \& Olszanska 2003).

\section{Potential mechanisms determining the outcome of polyspermy}

In this study, we discuss some candidate reproductive, cellular, and developmental mechanisms that may explain variation in the effects of polyspermy on centrosome dynamics across taxa. We have attempted to organize these ideas hierarchically, from broad scale (e.g. mode of fertilization) to finer scale (e.g. origin of mitotic spindles). We stress that there has been no focused research aimed at explicitly elucidating mechanisms underlying variance in the developmental outcome of polyspermy, so our discussion simply serves as a guide for highlighting questions that can be addressed by future research.

\section{Modes of fertilization}

One striking feature of the taxonomic distribution of the three types of polyspermy is the broad association with fertilization mode. While both internally and externally fertilizing taxa exhibit pathological polyspermy, instances of non-pathological polyspermy nearly exclusively only occur in taxa with internal fertilization (with the sole known exception of a ctenophore, B. ovata). Why would the mode of fertilization be associated with the outcome of polyspermy? Are there any differences between general reproductive biology and/or development (via microtubule dynamics) in externally fertilizing taxa with pathological, compared with internally fertilizing taxa having non-pathological, polyspermy? Are there similarities in external and internal fertilizing taxa that exhibit pathological polyspermy in how multiple sperm asters are mismanaged? What relevant cellular differences are there between internally fertilizing taxa displaying pathological compared with non-pathological polyspermy?

\section{Egg size}

Urodeles, reptiles, birds, and insects are different from other taxa in that they possess large yolky eggs and exhibit no blocks to polyspermy (for reviews see Tarín (2000) and Wong \& Wessel (2005)). Perhaps such eggs cannot support rapid electrical and ionic mediated mechanisms to block polyspermy, or perhaps their sheer size means that blocks of this nature are impractical. These taxa also exhibit non-pathological polyspermy. So perhaps taxa with large yolky eggs have evolved intracellular or cytological blocks that function in a localized way to suppress the action of supernumerary sperm. An alternative hypothesis is that large numbers of sperm may be necessary to ensure karyogamy (Birkhead et al. 1994) or otherwise provide paternal factors not fulfilled by a single sperm, to the large yolky egg during fertilization. In birds, the germinal disk, the area in which fusion ultimately occurs, is a relatively small area. In chickens and turkeys, the probability of fertilization is $100 \%$ when six or more sperm penetrate the inner perivitelline layer (IPVL) over the germinal disk, but this is reduced to $50 \%$ with only three penetrating sperm (Bramwell et al. 1995, Wishart 1997). This pattern supports the idea that polyspermy is necessary for successful karyogamy. However, given that 
the number of sperm entering a bird egg can range from 1 to $60+$, and that normal embryonic development can occur when only one sperm has entered the ovum (Wishart 1997) and many more than the minimum for $100 \%$ fertilization typically enter the egg, this cannot be the only explanation for polyspermy. While the correlation between egg size and non-pathological polyspermy is strong (Birkhead et al. 1994), this relationship is also unlikely to completely explain the outcome of polyspermy because it cannot explain both physiological and compensable polyspermy.

Egg size can influence the stochasticity of where and when sperm enter, and therefore potentially affect the timing of sperm aster formation and migration of potential MTOCs. Such differential timing may determine whether polyspermy is pathological. One study has examined the dynamics of sperm aster formation under various sperm entry conditions. Terada et al. (2009) used intracellular sperm injection to inject two human sperm into bovine eggs. They found no effect of either spatial or temporal variation of sperm injection on whether sperm asters formed, or the number of eggs forming zero, one, or two asters. Moreover, of those with one aster, the pattern of sperm injection did not influence whether it was the first or the second sperm that formed the aster. Thus, in such a heterologous artificial system, each sperm centrosome appeared to function independently of each other and form sperm asters. As bovines exhibit pathological polyspermy, it is assumed that, once formed, these asters interfere with each other resulting in aberrant first mitotic spindle assembly. No study has examined whether variation in the timing of sperm entry influences sperm centrosome dynamics in nonpathological polyspermic taxa. However, timing of MTOC migration and size of the centrosomes has been suggested to limit the pathological effects of multiple MTOCs in such taxa (see following section).

\section{First mitotic spindle assembly flexibility and parthenogenesis}

MTOCs for embryonic development appear to derive primarily from the sperm (see Karr (2001) for review) as a consequence of centriole inheritance from the sperm's basal body. Maternally derived centrosome inheritance could solve the problem of supernumerary sperm centrosome management and, indeed, such inheritance has been described in stick insects that exhibit physiological polyspermy (Marescalchi et al. 2002). However, maternal origin of the MTOC cannot be a universal mechanism to suppress the negative impact of supernumerary sperm, because mice have maternally derived MTOCs yet display pathological responses to polyspermy (Schatten et al. 1991). In addition, in some insects where the male normally donates a centriole, the egg cytoplasm can form centrioles if the male is prevented from doing so (i.e. parthenogenetic thelytokous reproduction), demonstrating that the egg has all the materials to form such a structure.

Thelytokous taxa must rely solely on egg contributions to organize microtubules. In parthenogenetic gynogenetic species - taxa where the sperm's typical and sole contribution is to trigger egg development - there are instances where a sperm-derived centrosome is used during embryogenesis (Schlupp 2005). Thus, both polyspermy and thelytoky require microtubule dynamics to be carefully managed for proper development; in pathological polyspermy the egg cannot do this, but in both non-pathological polyspermy taxa and in thelytokous taxa the egg successfully handles these microtubule issues. Thus, the de novo assembly of centrosomes in parthenogenetic taxa and in vertebrate systems in which the removal of a single mature cytoplasmic centriole elicits such assembly have been touted as excellent models for understanding centriole biogenesis (Loncarek \& Khodjakov 2009, Riparbelli et al. 2010). We argue that additional useful insights can be gained via polyspermy studies and suggest that research should address whether there are shared characteristics of microtubule management in thelytokous and nonpathological polyspermic taxa.

There are several Drosophila species that are either obligately or facultatively parthenogenetic (Suomalainen et al. 1987). In the facultative parthenogen, Drosophila mercatorum, parthenogenetic eggs assemble a few asters de novo and these concentrate in the anterior region of the egg near where the female pronucleus forms (Riparbelli \& Callaini 2003), whereas sexual strains do not exhibit de novo assembly. Riparbelli \& Callaini $(2003,2010)$ find only rare fusion between neighboring supernumerary sperm asters and suggest that there is some mechanism that ensures multiple asters are spaced to limit these fusions. Such a mechanism occurs in astral microtubules during intravitelline mitoses (Baker et al. 1993). Intriguingly, when eggs from parthenogenetic strains are fertilized, de novo assembly still occurs, a situation that generates multiple MTOCs. This pattern suggests that, in facultative parthenogenetic strains, the pathway of canonical centrosome assembly and de novo assembly dually exist whereas that duality is either not present in sexual strains or de novo assembly is somehow silenced. In Drosophila and mammals, the same proteins that are essential for canonical centrosome duplication are also necessary for de novo assembly (Bettencourt-Dias et al. 2005, Rodrigues-Martins et al. 2007) suggesting that silencing may be a feasible solution (see section 'A potential model' below).

Not necessarily mutually exclusive from silencing, as they could function at different developmental times, is the idea that timing of putative MTOC interactions may mediate whether multiple potential centrosomes result in developmental pathology. For example, in $D$. mercatorum, maternally derived asters are smaller than 
paternally derived ones, and therefore paternally derived asters are more likely to reach the female pronucleus, and establish interactions with the female chromatin, at an earlier stage than maternally derived asters (Riparbelli \& Callaini 2003). This situation is at least superficially similar to the formation of the principle sperm nucleus in C. pyrrhogaster that exhibits physiological polyspermy. Whether such differences in $D$. mercatorum are due to factors associated with the sperm basal body or egg cytoplasmic effects that influence microtubule nucleating dynamics is unknown. In some species, such as the surf clam (Spisula solidissima), paternal and maternal centrosomes appear to be distinguishable based on molecular markers that differentially regulate the nucleating dynamics of centrosomes deriving from each parent (Wu \& Palazzo 1999).

Another interesting case is that of the haplodiploid hymenoptera in which some eggs are haploid and develop as males and others are fertilized and develop into diploid females. In this study, as with facultatively parthenogenetic species, a single female produces eggs capable of proper development by either de novo aster formation (haploid) or using the sperm centrosome. Regardless of its ploidy state, at the end of meiosis II, haploid and diploid eggs have many cytoplasmically derived asters at the egg cortex periphery. However, the timing of migration of these cytoplasmic asters varies in haploid compared with diploid eggs. In fertilized eggs, migration of the female pronucleus toward the male pronucleus occurs before or concomitant with internal movement of the cytoplasmic asters and this spatial exclusion may be sufficient to allow the male and female pronucleus to fuse before any interference from competing MTOCs (Tram \& Sullivan 2000). Once fused, Tram \& Sullivan (2000) suggested that astral microtubule nucleation is adequate in preventing supernumerary centrosome attachment. These centrosome dynamics are different from the facultatively parthenogenetic $D$. mercatorum in which only a few maternally derived centrosomes, limited in their anterior location, are formed and in which the mechanism to produce such centrosomes are different (Ferree et al. 2006).

What do these developmental centrosome patterns in parthenogenetic taxa tell us about non-pathological polyspermy? To what extent are organisms that can reproduce parthenogenetically capable of exhibiting non-pathological polyspermy? Unfortunately, large datasets compiling the incidence of parthenogenesis and polyspermy are unavailable, probably because of the difficulty in studying either process. Therefore, only anecdotal evidence can be reported, although such cases may serve to stimulate further study. For example, komodo dragons (Watts et al. 2006) and zebra finch (Schutt et al. 2008) have recently been found to be capable of parthenogenetic reproduction and, as reptiles and birds, respectively, also exhibit physiological polyspermy.

\section{First cleavage mitosis variation}

Development in pathological polyspermy is not 'aberrant' until cleavage begins and occurs as a consequence of improper/random mixing of maternal and paternal chromosomes, so perhaps there is something about cleavage that influences the developmental outcome of polyspermy. Three different modes of first cleavage mitosis following fertilization have been described in animals (Kawamura 2001): sea urchin type, Ascaris type, and gonomeric (Fig. 4). These types differ in when the maternal and paternal chromosomes are intermixed during the first mitotic division of the embryo. In the sea urchin model, karyogamy occurs shortly before syngamy (fusion of the gametes). In the Ascaris model, parental chromosomes are intermixed on the first mitotic spindle (karyogamy) and as such, the mitotic apparatus has formed between a divided sperm aster and therefore true syngamy does not occur. In gonomery, similar to the Ascaris type, true syngamy does not occur and karyogamy does not occur until telophase. Longo (1973) has demonstrated that the eggs of most organisms exhibit an Ascaris type of first cleavage mitosis and that the sea urchin type can be experimentally altered to that of an Ascaris type. Thus, there may be some flexibility in the patterns of maternal and paternal integration during first cleavage. However, there are fundamental differences between the Ascaris/sea urchin type(s) compared with gonomery. To accomplish development following a gonomeric pattern, mitotic spindles are independently formed in each pronuclei rather than the mitotic apparatus being formed between the central bodies as occurs in both the sea urchin and Ascaris types of first cleavage mitosis. Could this difference in the way paternal and maternal chromosomes are managed during mitotic division explain the outcome of polyspermy and the ability of the egg to cope with supernumerary sperm? Perhaps, but only partially, as gonomery appears to occur only in the Arthropoda (some taxa of which exhibit either physiological or compensable polyspermy) yet other taxa with nonpathological polyspermy exhibit either the sea urchin or the Ascaris type cleavage patterns. Moreover, both non-pathological and pathological taxa can have either the sea urchin or the Ascaris type cleavage patterns. However, given that polyspermy is not pathological until cleavage, future studies may benefit from examining these patterns more closely.

\section{A potential model?}

The presence of multiple centrosomes is not a problem for taxa exhibiting non-pathological polyspermy. Likewise, tumor cells can suppress any extra chromosomes and form a normal bipolar spindle (Quintyne et al. 2005, Gergely \& Basto 2008, Kwon et al. 2008). It has been suggested for cancer cells that suppression could 
Invertebrates

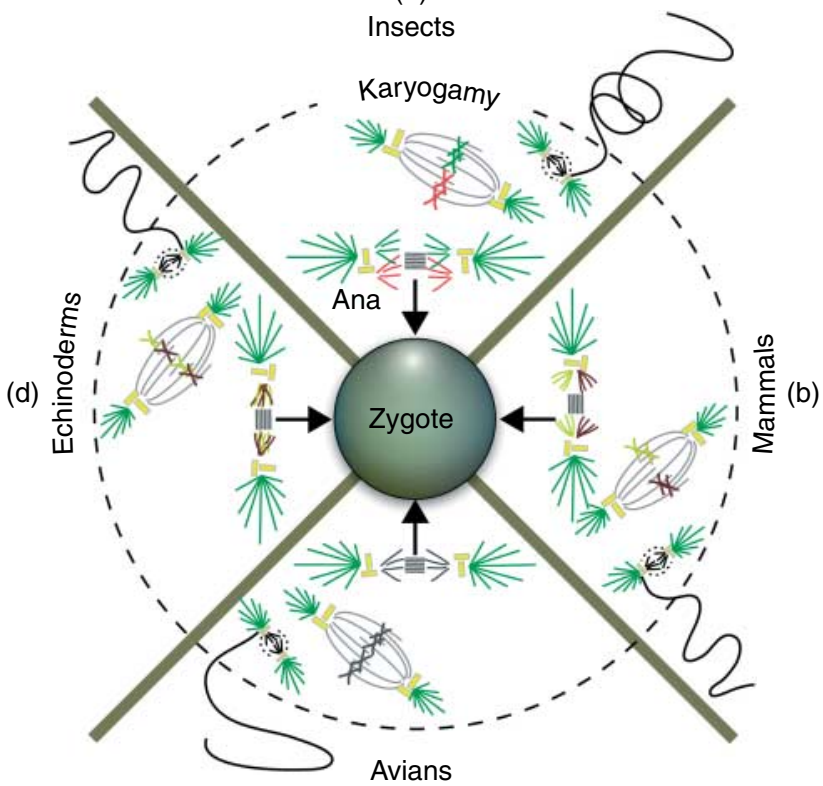

(c)

Vertebrates
Figure 4 Modes of first cleavage mitosis. As indicated, each pie sector represents one of the four major animal groups. The diagram depicts events immediately preceding karyogamy and ending in formation of a diploid zygote. When read inwards toward the center labeled 'zygote' (arrows), each sector details events leading to formation of the first mitotic division. Differences in coloration and details of the elements in each sector relate to known variation in specific processes during fertilization as discussed in the text. Sperm are represented by various elongated or spheroidal structures (sperm nuclei) attached to thin lines (sperm axonemes) via the basal body (yellow rectangles). Microtubules are shown as green lines, centrioles by paired yellow rectangles. The outermost structures, the extracellular egg matrix, where mammal, avian and echinoderm sperm interact initially with sperm are not shown. The ultimate result of fertilization is the formation of the zygote as indicated by the innermost circle. The processes of karyogamy (inside dashed line) are i) completion of DNA replication and chromatin condensation, ii) mitotic spindle formation and alignment of chromosomes on the metaphase plate, and iii) anaphase movement and completion of mitosis to form the first two zygotic nuclei. (a) Insects display an unusual form of karyogamy (gonomery) where the two pronuclei form physically distinct mitotic spindles (not shown) that then fuse to form a common spindle on which the parental chromosomes remain physically separated on the metaphase plate and remain separated during anaphase (ana). Parental chromosomes mix during telophase forming the diploid zygote. (b) Mammals - during karyogamy the parental chromosomes remain physically distinct on a common metaphase spindle and remain so throughout anaphase. Unlike insects, parental separation of genetic material continues until at least the fourth cell division (32-cell stage). (c) Avians - a single sperm enters the GD and fuses with the female pronucleus and the supernumerary sperm (SS) degenerate. The nature of karyogamy, nuclear fusion and chromosome behavior are less well known. (d) Echinoderms - during karyogamy the fused pronuclei form a common metaphase plate where parental chromosomes are mixed and indistinguishable by completion of the first mitosis. Adapted, with permission, from Karr TL Swanson WJ \& Snook RR 2009 The evolutionary significance of variation in sperm-egg interactions. In Sperm Biology: An Evolutionary Perspective, pp 305-365. Eds TR Birkhead, DJ Hosken, S Pitnick. London: Academic Press. CO2009 Elsevier. occur via either silencing the extra centrosomes or by coalescing them into only two functional spindle poles (Brinkley 2001). Evidence mostly supports coalescence in mammals (Brinkley 2001, Quintyne et al. 2005, Kwon et al. 2008) and it would be interesting to see if coalescence can occur in polyspermic eggs and, if so, under what conditions. In Drosophila, however, polyspermic eggs, which are not pathological, do not appear to coalesce the multiple centrosomes (Riparbelli \& Calliani 2010), instead, two different mechanisms appear to function to limit the negative developmental impact of multiple centrosomes (Basto et al. 2008).

First, bipolarity in Drosophila cells with multiple centrosomes can be restored by slowing down mitosis. This involves the spindle assembly checkpoint and kinesin/ $\mathrm{Ncd}$, to allow clustering of centrosomes to form two dominant poles that assemble the bipolar mitotic spindle. It would be informative to know whether polyspermic eggs are developmentally delayed due to a slowing of mitosis. Secondly, centrosomes do not always coalesce and extra centrosomes appear to be gradually inactivated by an unknown process, but perhaps due to competition for limiting/localized PCM or mitotic spindle supplies. In Drosophila, CycB and MPF activity do not oscillate in early development (Edgar et al. 1994), although there is local СусB degradation by the mitotic spindle (Huang \& Raff 2002, Raff et al. 2002). Polyspermy in Drosophila is compensable. In contrast, in sea urchin and frog embryos, the first 12 mitotic cycles are driven by a cytoplasmic clock causing periodic degradation of the $\mathrm{CycB}$ subunit of MPF and resultant nuclear divisions (Hara et al. 1980, Gerhart et al. 1984). Polyspermy is pathological in these species. Perhaps the reason why polyspermy is not pathological in Drosophila, but is in sea urchins and frogs, is that the more localized degradation of MPF could prevent potential MTOCs from forming spindles in the former but in the latter MPF signals are consistent across the egg therefore affecting all potential MTOCs similarly.

\section{Evolutionary causes and consequences of polyspermy}

To an extent, in the above sections, we have taken an evolutionary approach in thinking about the mechanisms that may generate variation in the outcome of polyspermy; that is, how traits are distributed across taxa exhibiting either pathological or non-pathological polyspermy. For a full understanding of variation in the outcome of polyspermy, a more detailed evolutionary perspective is required. Such evolutionary considerations may make it possible to predict when polyspermy will be pathological or not and may guide efforts to 
uncover common mechanisms controlling the fate of supernumerary sperm.

Polyspermy should be costly for females in that: a) it reduces their fitness through the loss of viable embryos when it is pathological, b) investment by females in external blocks to polyspermy could reduce fitness if such blocks are too efficient thereby preventing fertilization, and $\mathrm{c}$ ) producing the blocking machinery requires resources that could otherwise be spent on producing more eggs. Because of these costs, we expect females to be under selection to optimize their responses to reduce the risk of polyspermy. However, males are expected to be under strong selection to out-compete rival males in the race for fertilizations, and to produce both large numbers of sperm and sperm that are very efficient at entering ova (Parker 1984). Collateral damage from such sperm competition for fertilization may be pathological polyspermy. Thus, females will be selected to reduce the effects of polyspermy while males indirectly may be fostering polyspermy; such differences in the fertilization optimum between the sexes can generate conflict between the sexes that may drive the (co)evolution of male and female reproductive traits (Rice 1996, Levitan 2006, Moy et al. 2008, Hosken et al. 2009). For example, incubation in oviductal fluid of in vitro matured pigs eggs increased the incidence of monospermy during IVF. This increase was likely due to the oviductal fluid causing the zona pellucida (ZP) to become resistant to proteolytic digestion and decreased ZP-sperm binding (Coy et al. 2008a). Such oviductal-acquired ZP resistance occurs in several mammal species and does not appear to be species-specific (Coy et al. 2008a, 2008b).

The outcome of sexual conflict on the evolution of male and female traits can be complicated (Gavrilets et al. 2001, Moore \& Pizzari 2005, Rowe et al. 2005), but we suggest that, similar to female responses to other types of sexual conflict such as mating rate (Rowe et al. 2005), selection generated through polyspermy could elicit one of the two evolutionary responses by females. That is, females could evolve either increased tolerance to polyspermy via decreasing their sensitivity to it, or alternatively, females could evolve increased resistance to polyspermy via increasing their threshold to it. Such a dichotomy in female response (sensitivity vs threshold) would elicit the two observed evolutionary outcomes to polyspermy: polyspermy would be pathological when females resist it but, if overwhelmed, then negative fitness consequences occur, and polyspermy would be non-pathological if females decrease their sensitivity to polyspermy such that, when it occurs, negative fitness consequences are mitigated. If increased resistance evolves, then females should invest in polyspermy preventing mechanisms, whereas if increased tolerance evolves, then females should invest in centrosome management when supernumerary sperm enter eggs.

Previous sexual conflict models over mating rate suggest that only threshold responses (i.e. evolving increased resistance) will generate on-going malefemale arms races (Rowe et al. 2005). In the context of polyspermy, as females evolve better blocks to polyspermy, this then selects for males to evolve more penetrative sperm and fertilize ova before rivals do, which then again generates polyspermy which then selects for better blocks and so on. Empirical data to test such patterns associated with conflict over fertilization rate are scarce. However, work on sea urchins is consistent with this broad scenario (Levitan 1993, Levitan et al. 2007). For example, eggs from species in which high rates of fertilization can occur at relatively low sperm concentrations are more susceptible to polyspermy whereas eggs from species requiring relatively greater sperm concentrations are more resistant to polyspermy (Levitan et al. 2007). In addition, correlational evidence suggests that rapid evolution of gamete recognition proteins have occurred in many marine invertebrates and mammals (Swanson \& Vacquier 2002) that exhibit pathological polyspermy, but not in birds that experience physiological polyspermy (Calkins et al. 2007, Berlin et al. 2008). Thus, whether females change their sensitivity or threshold (Rowe et al. 2005) to polyspermy may explain why we observe different developmental outcomes to polyspermy. Future work should determine whether the threshold or sensitivity response to polyspermy by females is more evolutionarily labile and under what conditions such evolution will occur. Research using broadcast spawners that exhibit pathological polyspermy suggests that thresholds may have evolved in that group (Levitan et al. 2007) but such research has not been performed in taxa exhibiting non-pathological polyspermy.

While many of the evolutionary ideas about polyspermy result from it being a negative byproduct of sperm competition, it also seems possible that malemale competition could directly select for polyspermy. That is, when sperm from one male meets an ovum already penetrated by rival spermatozoa, selection could favor the entry of the second sperm because it causes developmental failure ( $\mathrm{R}$ Johnston, personal communications). This selfish behavior would require some means of ensuring only eggs entered by rival sperm were 'attacked' (on average) and possibly requires some local 'non-egg' competition between the offspring of competing males. Such behavior would obviously not be in the interest of females who could then respond by altering their threshold or sensitivity to polyspermy. The extent to which such a phenomenon can explain the evolution of polyspermy requires theoretical exploration.

\section{Conclusions}

We have described aspects of reproductive and developmental biology that vary across taxa exhibiting different outcomes to polyspermy, suggested some candidate 
proximate mechanisms that may explain this variation, and outlined how sperm competition and sexual conflict over fertilization may contribute to variation in the fitness consequences of polyspermy. Our goal is to facilitate the mechanistic and evolutionary understanding of why polyspermy is detrimental in some, but not all, taxa. Comparing centrosome dynamics that differ between taxa exhibiting pathological and non-pathological polyspermy will help to uncover the developmental mechanisms that buffer some organisms against developmental failure due to excess centrosomes. Such work may inform studies of human health issues, such as cancer and aging, in which excess centrosomes have been implicated. Moreover, understanding the selective factors that drives fitness variation in the outcome of polyspermy may also help point to candidate mechanisms that promote or constrain centrosome management.

From a proximate perspective, understanding the mechanisms that control centrosome management can be facilitated by comparisons between both pathological and non-pathological groups and between taxa exhibiting the two different forms of non-pathological polyspermy (physiological and compensable). While it is much too soon to be able to provide definitive explanations for the variation in polyspermy outcomes, mostly because too little research on potential key traits have been performed, we outline avenues available for immediate advance, and these should be investigated.

Evolutionary perspectives that may facilitate understanding polyspermy variation are hampered because we do not have an adequate knowledge of the evolutionary distribution of the outcomes. Whether direct or indirect, male-male competition and malefemale (sexual) conflict have been suggested to be key agents in the evolution of polyspermy (Rice \& Holland 1997). We propose that sexual conflict arising through pathological polyspermy may result either in increased resistance to or increased tolerance of supernumerary sperm, with the former potentially resulting in escalating arms races between the sexes. Current limited data supports such an interpretation. Where compensable polyspermy is on this evolutionary response scale is unknown, but it could be an evolutionary intermediate between pathological and physiological polyspermy because selection against prevention (polyspermy resistance) is weakened by the tolerance of the egg to supernumerary sperm.

Both theoretical and empirical work is needed to clarify evolutionary and mechanistic responses to polyspermy. However, we can construct some links between potential mechanisms and evolutionary patterns of polyspermy based on our limited knowledge. For example, egg size has been shown to influence the risk of polyspermy (Levitan 1993) and species that experience higher risks of pathological polyspermy could (all else being equal) be selected to decrease egg (i.e. target) size. However, egg size must meet some minimum to support embryonic development and, in some species where the amount of yolk is critical to embryo survival, selection may not be able to decrease target size enough to decrease the risk of pathological polyspermy. Such a scenario would select for alternative female responses to polyspermy, perhaps decreasing sensitivity to polyspermy or evolving intracellular supernumerary sperm management tactics. One prediction of this model is that taxa with large yolky eggs would experience physiological polyspermy. While this is not an exclusive prediction, the prediction is broadly observed. Future research should work toward a complete phylogenetic analysis including mechanistic data to allow the development of additional hypotheses and to fully understand why we observe different outcomes of polyspermy.

Given that polyspermy is one of the most commonly observed 'abnormalities' in fertilization, it is surprising that both reproductive and evolutionary biologists have not taken advantage of the taxonomic variability in the outcome of polyspermy. Such variability permits understanding of both proximate and ultimate mechanisms controlling the fitness consequences of polyspermy. Rapid insights could be made if such studies are done cooperatively between reproductive and evolutionary biologists.

\section{Declaration of interest}

The authors declare that there is no conflict of interest that could be perceived as prejudicing the impartiality of the review.

\section{Funding}

R R Snook was supported by NERC.

\section{Acknowledgements}

We thank Rufus Johnston and Mike Ball for discussions about polyspermy, and Tim Birkhead and two anonymous reviewers for comments on an earlier draft.

\section{References}

Baker J, Theurkauf WE \& Schubiger G 1993 Dynamic changes in microtubule configuration correlate with nuclear migration in the preblastoderm Drosophila embryo. Journal of Cell Biology 122 113-121. (doi:10.1083/jcb.122.1.113)

Basto R, Lau J, Vinogradova T, Gardiol A, Woods CG, Khodjakov A \& Raff JW 2006 Flies without centrioles. Cell 125 1375-1386. (doi:10. 1016/j.cell.2006.05.025)

Basto R, Brunk K, Vinadogrova T, Peel N, Franz A, Khodjakov A \& Raff JW 2008 Centrosome amplification can initiate tumorigenesis in flies. Cell 133 1032-1042. (doi:10.1016/j.cell.2008.05.039) 
Berlin S, Qu L \& Ellegren H 2008 Adaptive evolution of gamete-recognition proteins in birds. Journal of Molecular Evolution 67 488-496. (doi:10. 1007/s00239-008-9165-6)

Bettencourt-Dias M, Rodrigues-Martins A, Carpenter L, Riparbelli M, Lehmann L, Gatt MK, Carmo N, Ballous F, Callaini G \& Glover DM 2005 SAK/PLK4 is required for centriole duplication and flagella development. Current Biology 15 2199-2207. (doi:10.1016/j.cub.2005.11.042)

Birkhead TR, Sheldon B \& Fletcher F 1994 A comparative study of spermegg interactions in birds. Journal of Reproduction and Fertility $\mathbf{1 0 1}$ 353-361. (doi:10.1530/jrf.0.1010353)

Blount M 1909 The early development of the pigeon's egg, with special reference to polyspermy and the origin of the periblast nuclei. Journal of Morphology 20 1-64. (doi:10.1002/jmor.1050200102)

Boveri 1901 Zellen-Studien: Über die Natur der Centrosomen, vol 28, pp 1-220. Jena. Germany: Fisher Z Med Naturw

Bramwell RK, Marks HL \& Howarth B 1995 Quantitative determination of spermatozoa penetration of the perivitelline layer of the hen's ovum as assessed on oviposited eggs. Poultry Science 74 1875-1883.

Breed WG, Simerly C, Navara CS, VandeBerg J \& Schatten G 1994 Microtubule configurations in oocytes, zygotes, and early embryos of a marsupial, Monodelphis domestica. Developmental Biology 164 230-240. (doi:10.1006/dbio.1994.1194)

Brinkley BR 2001 Managing the centrosome numbers game: from chaos to stability in cancer cell division. Trends in Cell Biology 11 18-21. (doi:10. 1016/S0962-8924(00)01872-9)

Calkins JD, El-Hinn D \& Swanson WJ 2007 Adaptive evolution in an avian reproductive protein: ZP3. Journal of Molecular Evolution 65 555-563. (doi:10.1007/s00239-007-9034-8)

Calliani G \& Riparbelli MG 1996 Fertilization in Drosophila melanogaster: centrosome inheritance and organization of the first mitotic spindle. Developmental Biology 176 199-208. (doi:10.1006/dbio.1996.0127)

Calliani G, Riparbelli MG \& Dallai R 1999 Centrosome inheritance in insects: fertilization and parthenogenesis. Biology of the Cell 91 355-366. (doi:10.1016/S0248-4900(99)80096-3)

Carré D \& Sardet C 1984 Fertilization and early development in Beroe ovata. Developmental Biology 105 188-195. (doi:10.1016/0012-1606 (84)90274-4)

Carré D, Rouvière C \& Sardet C 1991 In vitro fertilization in ctenophores: sperm entry, mitosis, and the establishment of bilateral symmetry in Beroe ovata. Developmental Biology 147 381-391. (doi:10.1016/00121606(91)90296-F)

Counce SJ 1959 Spermatozoa in Drosophila eggs. Drosophila Information Service 33 127-128.

Coy P, Cánovas S, Mondéjar I, Saavedra MD, Romar R, Grullón L, Matás C \& Avilés M 2008a Oviduct-specific glycoprotein and heparin modulate sperm-zona pellucid interaction during fertilization and contribute to the control of polyspermy. PNAS 105 15809-15814. (doi:10.1073/pnas. 0804422105)

Coy P, Grullón L, Cánovas S, Romar R, Matás C \& Avilés M 2008b Hardening of the zona pellucida of unfertilized eggs can reduce polyspermic fertilization in the pig and cow. Reproduction 135 19-27. (doi:10.1530/REP-07-0280)

Degrugillier ME \& Leopold RA 1976 Ultrastructure of sperm penetration of house fly eggs. Journal of Ultrastructural Research 56 312-325. (doi:10. 1016/S0022-5320(76)90006-X)

Edgar BA, Sprenger F, Duronio RJ, Leopold P \& O'Farrell PH 1994 Distinct molecular mechanism regulate cell cycle timing at successive stages of Drosophila embryogenesis. Genes and Development 8 440-452. (doi:10.1101/gad.8.4.440)

Elinson RP 1986 Fertilization in amphibian: the ancestry of the block to polyspermy. International Review of Cytology 101 59-100. (doi:10. 1016/S0074-7696(08)60246-6)

Fechheimer NS 1981 Origins of heteroploidy in chick embryos. Poultry Science 60 1365-1371.

Ferree PM, McDonald K, Fasulo B \& Sullivan W 2006 The origin of centrosomes in parthenogenetic hymenopteran insects. Current Biology 16 801-817. (doi:10.1016/j.cub.2006.03.066)

Forstmeier W \& Ellegren H 2010 Trisomy and triploidy are sources of embryo mortality in the zebra finch. Proceedings of the Royal Society of London, Series B 277 2655-2660. (doi:10.1098/rspb.2010.0394)
Gavrilets S, Arnqvist G \& Friberg U 2001 The evolution of female mate choice by sexual conflict. Proceedings of the Royal Society of London, Series B 268 531-539. (doi:10.1098/rspb.2000.1382)

Gergely F \& Basto R 2008 Multiple centrosomes: together they stand, divided they fall. Genes and Development 22 2291-2296. (doi:10.1101/ gad.1715208)

Gerhart J, Wu M \& Kirschner MW 1984 Cell cycle dynamics of an M-phase cytoplasmic factor in Xenopus laevis oocytes and eggs. Journal of Cell Biology 98 1247-1255. (doi:10.1083/jcb.98.4.1247)

Hara K, Tydeman P \& Kirschner M 1980 A cytoplasmic clock with the same period as the division cycle in Xenopus eggs. PNAS 77 462-466. (doi:10. 1073/pnas.77.1.462)

Hildreth PE \& Lucchesi JC 1963 Fertilization in Drosophila I. Evidence for the regular occurrence of monospermy. Developmental Biology 6 262-278. (doi:10.1016/0012-1606(63)90015-0)

Hosken DJ, Martin OY, Wigby S, Chapman T \& Hodgson DJ 2009 Sexual conflict and reproductive isolation in flies. Biology Letters 5 697-699. (doi:10.1098/rsbl.2009.0066)

Huang JY \& Raff JW 2002 The dynamic localisation of the Drosophila APC/C: evidence for the existence of multiple complexes that perform distinct functions and are differentially localised. Journal of Cell Science 115 2847-2856.

Huettner AF 1924 Maturation and fertilization in Drosophila melanogaster. Journal of Morphology 39 249-265. (doi:10.1002/jmor.1050390108)

Hunter RHF 1998 Polyspermy. In Encyclopedia of Reproduction, vol 3, pp 930-937. Eds E Knobil \& JD Neill. London: Academic Press.

Iwamatsu T 2000 Fertilization in fishes. In Fertilization in Protozoa and Metazoan Animals, pp 89-146. Eds JJ Tarín\& A Cano. Berlin: Springer.

Iwao Y 2000 Mechanisms of egg activation and polyspermy block in amphibians and comparative aspects with fertilization in other vertebrates. Zoological Science 17 699-709. (doi:10.2108/zsj.17.699)

Iwao Y, Murakawa T, Yamaguchi J \& Yamashita M 2002 Localization of $\gamma$-tubulin and cyclin B during early cleavge in physiologically polyspermic newt eggs. Development, Growth \& Differentiation 44 489-499. (doi:10.1046/j.1440-169X.2002.00661.x)

Jordan EO 1893 The habits and development of the newt (Diemyctylus viridescens). Journal of Morphology 8 269-366. (doi:10.1002/jmor. 1050080203)

Jura C \& Krzysztofowicz A 1992 Initiation of embryonic development in Tetrodontophora bielanensis Waga) (Collembola) eggs: meiosis, polyspermy, union of gametes and the first cleavage. International Journal of Insect Morphology and Embryology 21 87-94. (doi:10.1016/ 0020-7322(92)90007-A)

Karr TL 1991 Intracellular sperm/egg interactions in Drosophila: a threedimensional structural analysis of a paternal product in the developing egg. Mechanisms of Development 34 101-112. (doi:10.1016/09254773(91)90047-A)

Karr TL 1996 Paternal investment and intracellular sperm-egg interactions during and following fertilization in Drosophila. Current Topics in Developmental Biology 34 89-115. (doi:10.1016/S0070-2153(08) 60709-7)

Karr TL 2001 Centrosome inheritance: a central 'in-egg-ma' solved? Current Biology 11 R21-R24. (doi:10.1016/S0960-9822(00)00037-3)

Karr TL \& Pitnick S 1996 The ins and outs of fertilization. Nature 379 405-406. (doi:10.1038/379405a0)

Karr TL, Swanson WJ \& Snook RR 2009 The evolutionary significance of variation in sperm-egg interactions. In Sperm Biology: An Evolutionary Perspective, pp 305-365. Eds TR Birkhead, DJ Hosken\& S Pitnick. London: Academic Press.

Kawamura N 2001 Fertilization and the first cleavage mitosis in insects. Development, Growth \& Differentiation 43 343-349. (doi:10.1046/ j.1440-169x.2001.00584.x)

Kwon M, Godinho SA, Chandhok NS, Ganem NJ, Azioune A, Thery M \& Pellman D 2008 Mechanisms to suppress multipolar divisions in cancer cells with extra centrosomes. Genes and Development 22 2189-2203. (doi:10.1101/gad.1700908)

Landmann F, Orsi GA, Loppin B \& Sullivan W 2009 Wolbachia-mediated cytoplasmic incompatibility is associated with impaired histone deposition in the male pronucleus. PLoS Pathogens 5 e1000343. (doi:10.1371/journal.ppat.1000343) 
Laubichler MD \& Davidson EH 2008 Boveri's long experiment: sea urchin mergones and the establishment of the role of nuclear chromosomes in development. Developmental Biology 314 1-11. (doi:10.1016/j.ydbio. 2007.11.024)

Levitan DR 1993 The importance of sperm limitation to the evolution of egg size in marine invertebrates. American Naturalist 141 517-536. (doi:10.1086/285489)

Levitan DR 2006 The relationship between egg size and fertilization success in broadcast-spawning marine invertebrates. Integrative and Comparative Biology 46 298-311. (doi:10.1093/icb/icj025)

Levitan DR, terHorst CP \& Fogarty ND 2007 The risk of polyspermy in three congeneric sea urchins and its implications for gametic incompatibility and reproductive isolation. Evolution 61 2007-2014. (doi:10.1111/j. 1558-5646.2007.00150.x)

Linhart O \& Kudo S 1997 Surface ultrastructure of paddlefish eggs before and after fertilization. Journal of Fish Biology 51 573-582. (doi:10.1111/ j.1095-8649.1997.tb01513.x)

Loncarek J \& Khodjakov A 2009 Ab ovo or de novo? Mechanisms of centriole duplication Molecules and Cells 27 135-142. (doi:10.1007/ s10059-009-0017-z)

Long CR, Pinto-Correia C, Duby RT, Ponce de Leon FA, Boland MP, Roche JF \& Robl JM 1993 Chromatin and microtubule morphology during the first cell cycle in bovine zygotes. Molecular Reproduction and Development 36 23-32. (doi:10.1002/mrd.1080360105)

Longo FJ 1973 Fertilization: a comparative ultrastructural review. Biology of Reproduction 9 140-215.

Mantovani B \& Scali V 1992 Hybridogenesis and androgenesis in the stickinsect Bacillus rossius-grandii benazzii (Insecta Phasmatodea). Evolution 46 783-796. (doi:10.2307/2409646)

Marescalchi O, Zauli C \& Scali V 2002 Centrosome dynamics and inheritance in related sexual and parthenogenetic Bacillus (Insecta Phasmatodea). Molecular Reproduction and Development 63 89-95. (doi:10.1002/mrd.10177)

Miller RC, Fechheimer NS \& Jaap RG 1976 Distribution of karyotype abnormalities in chick embryo sibships. Biology of Reproduction 14 549-560. (doi:10.1095/biolreprod14.5.549)

Moore AJ \& Pizzari T 2005 Quantitative genetic models of sexual conflict based on interacting phenotypes. American Naturalist 165 S88-S97. (doi:10.1086/429354)

Moy GW, Springer SA, Adams SL, Swanson WJ \& Vacquier VD 2008 Extraordinary intraspecific diversity in oyster sperm bindin. PNAS 105 1993-1998. (doi:10.1073/pnas.0711862105)

Navara CS, Frist NL \& Schatten G 1994 Microtubule organization in the cow during fertilization, polyspermy, parthenogenesis, and nuclear transfer: the role of the sperm aster. Developmental Biology 162 29-40. (doi:10.1006/dbio.1994.1064)

Oppel A 1892 Die Befruchtung des Reptilieneies. Archiv für Mikroskopische Anatomie 39 215-290. (doi:10.1007/BF02961531)

Parker GA 1984 Sperm competition and the evolution of mating strategies. In Sperm Competition and the Evolution of Animal Mating Systems, pp 1-60. Ed. RL Smith. London: Academic Press.

Perry MM 1987 Nuclear events from fertilization to the early cleavage stages in the domestic fowl (Gallus domesticus). Journal of Anatomy $\mathbf{1 5 0}$ 99-109.

Quintyne NJ, Reing JE, Hoffelder DR, Gollin SM \& Saunders WS 2005 Spindle multipolarity is prevented by centrosomal clustering. Science 307 127-129. (doi:10.1126/science.1104905)

Raff JW, Jeffers K \& Huang JY 2002 The roles of Fzy/Cdc20 and Fzr/Cdh1 in regulating the destruction of cyclin B in space and time. Journal of Cell Biology 157 1139-1149. (doi:10.1083/jcb.200203035)

Rice WR 1996 Sexually antagonistic male adaptation triggered by experimental arrest of female evolution. Nature 361 232-234. (doi:10. 1038/381232a0)

Rice WR \& Holland B 1997 The enemies within: intergenomic conflict, interlocus contest evolution (ICE), and the intraspecific red queen. Behavioral Ecology and Sociobiology 41 1-10. (doi:10.1007/s00 2650050357)

Riemann JG 1965 The development of eggs of the screw wormfly Cochliomyia horninivorax (Coquerel) (Diptera: Calliphhoridae) to the blastoderm stage as seen in whole-mount preparations. Biological Bulletin 129 329-339. (doi:10.2307/1539849)
Riparbelli MG \& Callaini G 2003 Drosophila parthenogenesis: a model for de novo centrosome assembly. Developmental Biology 260 298-313. (doi:10.1016/S0012-1606(03)00243-4)

Riparbelli MG \& Callaini G 2010 Detachment of the basal body from the sperm tail is not required to organize functional centrosomes during Drosophila embryogenesis. Cytoskeleton 67 251-258. (doi:10.1002/cm. 20440)

Riparbelli MG, Dallai R, Mercati D, Bu Y \& Callaini G 2010 Centriole symmetry: a big tale from small organisms. Cell Motility and the Cytoskeleton 66 1100-1105. (doi:10.1002/cm.20417)

Rodrigues-Martins A, Riparbelli M, Callaini G, Glover DM \& BettencourtDias M 2007 Revisiting the role of the mother centriole in centriole biogenesis. Science 316 1046-1050. (doi:10.1126/science. 1142950)

Rothschild L 1956 Polyspermy. Quarterly Review of Biology 29 332-342.

Rowe L, Cameron E \& Day T 2005 Escalation, retreat, and female indifference as alternative outcomes of sexually antagonistic coevolution. American Naturalist 165 S5-S18. (doi:10.1086/429395)

Rückert J 1899 Die erste Entwicklung des Eies der Elasmobranchier. In Festschrift Zum Siebenzigsten Geburtstag von Carl von Kuppfer, pp 581-704. Jena: Gustav Fischer.

Sato M \& Tanaka-Sato H 2002 Fertilization, syngamy, and early embryonic development in the cricket Gryllus bimaculatus De Geer). Journal of Morphology 254 266-271. (doi:10.1002/jmor.10033)

Schatten G 1994 The centrosome and its mode of inheritance: the reduction of the centrosome during gametogenesis and its restoration during fertilization. Developmental Biology 165 299-335. (doi:10.1006/dbio. 1994.1256)

Schatten H 2008 The mammalian centrosome and its function. Histochemistry and Cell Biology 129 667-686. (doi:10.1007/s00418008-0427-6)

Schatten G, Simerly C \& Schatten H 1991 Maternal inheritance of centrosomes in mammals? Studies of parthenogenesis and polyspermy in mice PNAS 88 6785-6789. (doi:10.1073/pnas.88.15.6785)

Schlupp I 2005 The evolutionary ecology of gynogenesis. Annual Reviews of Ecology, Evolution and Systematics 36 399-417. (doi:10.1146/ annurev.ecolsys.36.102003.152629)

Schutt E, Hemmings N \& Birkhead TR 2008 Parthenogenesis in a passerine bird, the zebra finch, Taeniopygia guttata. Ibis 150 197-199. (doi:10. 1111/j.1474-919X.2007.00755.x)

Simerly C, Zoran SS, Payne C, Dominko T, Sutovsky P, Navara CS, Salisbury JL \& Schatten G 1999 Biparental inheritance of $\gamma$-tubulin during human fertilization: molecular reconstitution of functional zygotic centrosomes in inseminated human oocytes and in cell-free extracts nucleated by human sperm. Molecular Biology of the Cell $\mathbf{1 0}$ 2955-2969.

Snook RR \& Karr TL 1998 Only long sperm are fertilization-competent in six sperm-heteromorphic Drosophila species. Current Biology 8 291-294. (doi:10.1016/S0960-9822(98)70112-5)

Stepinska U \& Bakst MR 2007 Fertilization. In Reproductive Biology and Phylogeny of Birds: Phylogeny, Morphology, Hormones, Fertilization, vol 6A of series, pp 553-587. Ed. BGM Jamieson. Enfield, NH: Science Publishers.

Stepinska U \& Olszanska B 2003 DNase I and II present in avian oocytes; a possible involvement in sperm degradation at polyspermic fertilization. Zygote 11 35-42. (doi:10.1017/S0967199403001059)

Sun QY \& Schatten H 2007 Centrosome inheritance after fertilization and nuclear transfer in mammals. Somatic Cell Nuclear Transfer 591 58-71.

Suomalainen E, Saura A \& Lokki J 1987 Cytology and Evolution in Parthenogenesis, Boca Raton, FL: CRC Press.

Swanson WJ \& Vacquier VD 2002 The rapid evolution of reproductive proteins. Nature Reviews Genetics 3 137-144. (doi:10.1038/nrg733)

Tarín JJ 2000 Fertilization in protozoa and metazoan animals: a comparative overview. In Fertilization in Protozoa and Metazoan Animals, pp 277-314. Eds JJ Tarín\& A Cano. Berlin: Springer.

Terada Y, Hasegawa H, Ugajin T, Murakami T, Yaegashi N \& Koamura K 2009 Microtubule organization during human parthenogenesis. Fertility and Sterility 91 1271-1272. (doi:10.1016/j.fertnstert.2008.05.051)

Tiegs OW \& Murray FV 1938 Memoirs: the embryonic development of Calandra oryzae. Quarterly Journal of Microscopical Science $\mathbf{8 0}$ 159-284. 
Tram U \& Sullivan W 2000 Reciprocal inheritance of centrosomes in the parthenogenetic Hymenopteran Nasonia vitripennis. Current Biology 10 1413-1419. (doi:10.1016/S0960-9822(00)00795-8)

Waddington D, Gribbin C, Sterling RJ, Sang HM \& Perry MM 1998 Chronology of events in the first cell cycle of the polyspermic egg of the domestic fowl (Gallus domesticus). International Journal of Developmental Biology 42 625-628.

Wang W-H, Day BN \& Wu G-M 2003 How does polyspermy happen in mammalian oocytes? Microscopy Research and Technique 61 335-342. (doi:10.1002/jemt.10346)

Watts PC, Buley KR, Sanderson S, Boardman W, Ciofi C \& Gibson R 2006 Parthenogenesis in Komodo dragons. Nature 444 1021-1022. (doi:10. 1038/4441021a)

Wilson EB 1925 The Cell in Development and Inheritance. New York: Macmillan.

Wishart GJ 1997 Quantitative aspects of sperm:egg interaction in chickens and turkeys. Animal Reproduction Science 48 81-92. (doi:10.1016/ S0378-4320(97)00042-0)
Wishart GJ \& Horrocks AJ 2000 Fertilization in birds. In Fertilization in Protozoa and Metazoan Animals, pp 193-222. Eds JJ Tarín\& A Cano. Berlin: Springer.

Wong JL \& Wessel GM 2005 Defending the zygote: search for the ancestral animal block to polyspermy. Current Topics in Developmental Biology 72 1-151. (doi:10.1016/S0070-2153(05)72001-9)

Wu XY \& Palazzo RE 1999 Differential regulation of maternal vs paternal centrosomes. PNAS 96 1397-1402. (doi:10.1073/pnas.96.4. 1397)

Received 15 July 2011

First decision 15 August 2011

Revised manuscript received 15 September 2011

Accepted 28 September 2011 